Research Article

\title{
Dynamics in a Delayed Competition System on a Weighted Network
}

\author{
Yaohua Tong $(\mathbb{D}$ and Xiaoling Wang \\ School of Mathematics and Statistics, Shanxi Datong University, Shanxi, Datong 037009, China \\ Correspondence should be addressed to Yaohua Tong; sttongyh@sxdtdx.edu.cn
}

Received 10 October 2020; Revised 29 November 2020; Accepted 7 December 2020; Published 23 December 2020

Academic Editor: Stefan Balint

Copyright (c) 2020 Yaohua Tong and Xiaoling Wang. This is an open access article distributed under the Creative Commons Attribution License, which permits unrestricted use, distribution, and reproduction in any medium, provided the original work is properly cited.

In this paper, we study the stability of positive steady states in a delayed competition system on a weighted network, which does not satisfy the comparison principle appealing to classical competitive systems. By introducing some auxiliary equations and constructing proper contracting rectangles, we present some sufficient conditions on the stability of the unique positive steady state. Moreover, some numerical examples are given to explore the complex dynamics of this nonmonotone model, which implies the nontrivial roles of weights and time delays.

\section{Introduction}

Competition is one of the most universal phenomena in the natural world due to the limit resources including water and sunshine. When the amount of individuals is concerned in population dynamics, some differential systems modeling both interspecific and intraspecific competitions have been proposed and studied. For example, if only two competitive species are concerned in population dynamics, then one famous model is

$$
\left\{\begin{array}{l}
\frac{\mathrm{d} N_{1}}{\mathrm{~d} t}=r_{1} N_{1}\left(1-N_{1}-b_{1} N_{2}\right), \\
\frac{\mathrm{d} N_{2}}{\mathrm{~d} t}=r_{2} N_{2}\left(1-N_{2}-b_{2} N_{1}\right),
\end{array}\right.
$$

in which all the parameters are positive, and $N_{1}, N_{2}$ present the densities of two competitive species; we may refer to Murray [1], section 3.5, for the dynamics and biology background of (1). When multispecies and delayed effect [2-4] were considered, Faria [5] and Smith [6] studied the following delayed system:

$$
\frac{\mathrm{d} u_{i}(t)}{\mathrm{d} t}=r_{i} u_{i}(t)\left[1-\sum_{j=1}^{n} c_{i j} \int_{-\tau}^{0} u_{j}(t+s) \mathrm{d} \bar{\eta}_{i j}(s)\right],
$$

in which $i \in\{1,2, \ldots, n\}=: I, t>0$, all the parameters are positive, and $\bar{\eta}_{i j}(s)$ formulates the history delayed effect and satisfies

$$
\begin{aligned}
& \bar{\eta}_{i j}(s) \text { is nondecreasing on }[-\tau, 0], \\
& \bar{\eta}_{i j}(0)-\bar{\eta}_{i j}(-\tau)=1, \quad i, j \in I .
\end{aligned}
$$

In some cases, the spatial distribution of individuals must be considered. For example, to control the disease spreading, the spatial distribution of the infected is important. To model the spatial distribution of individuals, many reaction-diffusion systems have been established and studied; see [7-10]. In particular, we refer to $\mathrm{He}$ and $\mathrm{Ni}$ [11] and references cited therein for some results on the stability of different steady states in the corresponding reactiondiffusion system of (1). Moreover, when the spatial habitat is $\mathbb{R}$, Weinberger et al. [12] studied the invasion dynamics between the invader and the native in the corresponding 
diffusion model of (1). For the corresponding diffusion model of (2), Martin and Smith [13] proved the stability of steady states in the bounded domain. In the classical reaction-diffusion systems, it is necessary to assume that the density of individuals is continuous in both spatial and temporal variables. However, the spatial continuity is difficult to be satisfied due to the limitation of data in some cases. To overcome the deficiency, one recipe is to study the ordinary differential equations on a weighted network by considering finite patches as habitat of individuals; we may refer to Beretta et al. [14], Faria [5], Gao and Ruan [15], Liao and Lou [16], Mai et al. [17], Sun and Mai [18], Xu and Chen [19], and Zhang and Wang [20] for patch/network models with time delay.
Recently, Liu et al. [21] considered the competition dynamics of two competitors on a network or $m$ patches $(m>1$ and $m \in \mathbb{N})$; their model can be written as

$$
\left\{\begin{array}{l}
\frac{\mathrm{d} N_{1}^{j}}{\mathrm{~d} t}=\sum_{i=1}^{m} d_{i j}^{1}\left[N_{1}^{i}-N_{1}^{j}\right]+r_{1} N_{1}^{j}\left(1-N_{1}^{j}-b_{1} N_{2}^{j}\right), \\
\frac{\mathrm{d} N_{2}^{j}}{\mathrm{~d} t}=\sum_{i=1}^{m} d_{i j}^{2}\left[N_{2}^{i}-N_{2}^{j}\right]+r_{2} N_{2}^{j}\left(1-N_{2}^{j}-b_{2} N_{1}^{j}\right),
\end{array}\right.
$$

in which $j \in\{1,2, \ldots, m\}=: J, d_{i j}^{l}>0$ describes the movement between the $i$-th and $j$-th patches of the $l$-th species, which is called the weight between patches $i$ and $j$. In [18], Sun and Mai studied the following system on a network:

$$
\left\{\begin{array}{l}
\frac{\mathrm{d} N_{1}^{j}(t)}{\mathrm{d} t}=\sum_{i=1}^{m} d_{i j}^{1}\left[N_{1}^{i}\left(t-\tau_{1}\right)-N_{1}^{j}(t)\right]+r_{1} N_{1}^{j}(t)\left(1-N_{1}^{j}(t)-b_{1} N_{2}^{j}(t)\right) \\
\frac{\mathrm{d} N_{2}^{j}(t)}{\mathrm{d} t}=\sum_{i=1}^{m} d_{i j}^{2}\left[N_{2}^{i}\left(t-\tau_{2}\right)-N_{2}^{j}(t)\right]+r_{2} N_{2}^{j}(t)\left(1-N_{2}^{j}(t)-b_{2} N_{1}^{j}(t)\right)
\end{array}\right.
$$

in which $\tau_{1} \geq 0$ and $\tau_{2} \geq 0$ are time delays. A natural question is how to further study the dynamics of multicompetitive species with delayed effect on networks. In this paper, we study the following competition system with time delays on the network:

$$
\left\{\begin{array}{l}
\frac{\mathrm{d} u_{i}^{j}(t)}{\mathrm{d} t}=\sum_{l=1}^{m} d_{j l}^{i}\left[\int_{-\tau}^{0} u_{i}^{l}(t+s) d \rho_{j l}^{i}(s) \mathrm{d} s-u_{i}^{j}(t)\right]+r_{i} u_{i}^{j}(t)\left[1-\sum_{k=1}^{n} c_{i k} \int_{-\tau}^{0} u_{k}^{j}(t+s) \mathrm{d} \bar{\eta}_{i k}(s)\right], \quad t>0, \\
u_{i}^{j}(s)=v_{i}^{j}(s) \geq 0, \quad s \in[-\tau, 0],
\end{array}\right.
$$

in which all the parameters are positive, $j \in J, i \in I, t>0$, $u_{i}^{j}(t)$ denotes the population density of the $i$-th species on the $j$-th patch, and $\rho_{j l}^{i}$ satisfies

$$
\begin{aligned}
& \rho_{j l}^{i}(s) \text { is nondecreasing on }[-\tau, 0], \\
& \rho_{j l}^{i}(0)-\rho_{j l}^{i}(-\tau)=1, \quad i \in I, j, l \in J .
\end{aligned}
$$

That is, (6) is a coupled system of $m \times n$ functional differential equations. Evidently, there are $m$ patches in this model, the same species on different patches may move from one to another due to $d_{j l}^{i}>0$, and different species on the same patch compete each other. So, (6) is a competitive system on a weighted network or patch environment.

To compare the literature results with the complex phenomena in the real world, the stability of mathematical models is important since the disturbance is inevitable. Moreover, the controllability of many mathematical models in finite time is also important, which partly depends on the stability conditions of some states; see a number of examples about switched nonlinear systems [22-24]. Liu et al. [21] and Sun and Mai [18] studied the stability of different steady states of (4) and (5) by using the comparison principle and other techniques, which also include some applications in population dynamics. However, because of the intraspecific delayed effect, (6) does not satisfy the comparison principle of classical competitive systems, and the recipe in [21] does not work directly. Moreover, since there are $m \times n$ functional differential equations, the analysis of eigenvalues would be complex if we analyse the stability by the characteristic equation at the positive steady state, which may involve $m \times n$ transcendental equations. Therefore, to study the dynamics of (6), some other techniques are necessary.

Evidently, (6) may have several different steady states under proper conditions ((1) may have four steady states [1]), which reflect different biological sense in population dynamics. Coexistence of competitive species has been widely studied in many cases due to its important biological backgrounds on the biodiversity $[25,26]$. In this paper, by 
combining the technique in [21] with the contracting rectangles in [6], we study the stability of the positive steady state by giving some sufficient conditions. Moreover, we also show some numerical results to support our theoretical conclusions and present the complex dynamics of this system when our stability conditions do not hold, which implies the nontrivial role of time delays and partial degenerate weights.

The rest of this paper is organized as follows. In section 2 , we present some preliminaries on the stability via contracting rectangles. In Section 3, we show our main results on the stability of the unique positive steady state. Some numerical examples will be given in Section 4. Furthermore, we make a discussion in Section 5.

\section{Preliminaries}

In this paper, we use the standard partial ordering in $\mathbb{R}^{n}, n \in \mathbb{N}$. That is, if

$$
\begin{aligned}
& u=\left(u_{1}, u_{2}, \ldots, u_{n}\right), \\
& v=\left(v_{1}, v_{2}, \ldots, v_{n}\right) \in \mathbb{R}^{n},
\end{aligned}
$$

then

$$
u \leq v \text { if and only if } u_{i} \leq v_{i}, i \in\{1,2, \ldots, n\} .
$$

Moreover, $u<v$ implies $u \leq v$, but $u \neq v$, and $u \ll v$ implies $u_{i}<v_{i}, i \in\{1,2, \ldots, n\}$. $C\left(A, \mathbb{R}^{m}\right), A \subset \mathbb{R}^{n}$, denote the set of uniform continuous vector functions from $A$ to $\mathbb{R}^{n}$.

In this part, we recall the stability of the steady state by contracting rectangles [6]. Consider the functional differential system

$$
\left\{\begin{array}{l}
\frac{\mathrm{d} v_{i}(t)}{\mathrm{d} t}=f_{i}\left(v_{t}\right), \quad i \in\{1,2, \ldots, r\}=: R, t>0, \\
v_{i}(s)=v_{i}(s) \in C([-\tau, 0],[0, \infty)), i \in R, s \in[-\tau, 0],
\end{array}\right.
$$

in which $\tau>0$ is the delay, $v=\left(v_{1}, v_{2}, \ldots, v_{r}\right) \in \mathbb{R}^{r}$, and $f_{i}: C\left([-\tau, 0], \mathbb{R}^{r}\right) \longrightarrow \mathbb{R}$ satisfies the following assumptions:

(H1) There exists $E=\left(E_{1}, E_{2}, \ldots, E_{r}\right) \in \mathbb{R}^{r}$ with $E \gg 0$ such that $f_{i}(\widehat{0})=f_{i}(\widehat{E})=0, i \in R$, where $\widehat{r}$ denotes the constant-valued function in $C\left([-\tau, 0], \mathbb{R}^{r}\right)$.

$(\mathrm{H} 2)$ There exists a one-parameter family of ordered intervals given by

$$
\Sigma_{y}=[\widehat{a}(y), \widehat{b}(y)]
$$

such that $a(0) \leq E \leq b(0)$, and for $0 \leq y_{1} \leq y_{2} \leq 1$,

$$
\begin{aligned}
0 & \leq a(0) \leq a\left(y_{1}\right) \leq a\left(y_{2}\right) \leq a(1)=E=b(1) \\
& \leq b\left(y_{2}\right) \leq b\left(y_{1}\right) \leq b(0),
\end{aligned}
$$

where $a(y)$ and $b(y)$ are continuous in $y \in[0,1]$.

(H3) If $u \in C\left([-\tau, 0], \mathbb{R}^{r}\right)$ and $0 \leq v(s) \leq b(0)$ for $s \in[-\tau, 0]$, then

$$
f_{i}(v): C([-\tau, 0],[0, b(0)]) \longrightarrow \mathbb{R}
$$

is Lipschitz continuous in the sense of supremum norm for each $i \in R$.

(H4) $\Sigma_{y}$ is a strict contracting rectangle, namely, let

$$
\begin{aligned}
& a(y)=\left(a_{1}(y), a_{2}(y), \ldots, a_{r}(y)\right), \\
& b(y)=\left(b_{1}(y), b_{2}(y), \ldots, b_{r}(y)\right) .
\end{aligned}
$$
have

Then, for any $y \in(0,1)$ and $v_{0}(s) \in \Sigma_{y}, s \in[-\tau, 0]$, we

$$
\begin{aligned}
f_{i}\left(v_{0}\right) & >0\left(f_{i}\left(v_{0}\right)<0\right), \quad \text { if } u_{i}(0) \\
& =a_{i}(y)\left(u_{i}(0)=b_{i}(y)\right), i \in R .
\end{aligned}
$$

By contracting rectangles, we have the following conclusion [6].

Lemma 1. Assume that (H1)-(H4) hold. If $v_{i}(s) \in\left[a_{i}\left(y_{0}\right), b_{i}\left(y_{0}\right)\right] \quad$ for some $y_{0} \in(0,1)$ with $s \in[-\tau, 0], i \in R$, then

$$
\lim _{t \longrightarrow \infty} v_{i}(t)=E_{i}, \quad i \in R .
$$

\section{Main Results}

In this section, we study the long-time behavior of (6). We denote

$$
a_{i}=\bar{\eta}_{i i}(0)-\bar{\eta}_{i i}(0-), \quad i \in I,
$$

and set

$$
\begin{aligned}
\eta_{i i}(s) & =\left\{\begin{array}{l}
\bar{\eta}_{i i}(s), s \in[-\tau, 0), \\
\bar{\eta}_{i i}(0-), s=0,
\end{array}\right. \\
\eta_{i k}(s) & =\bar{\eta}_{i k}(s), \quad i, k \in I, i \neq k .
\end{aligned}
$$

In population dynamics, $a_{i}>0$ implies the existence of the instantaneous self-limitation effect.

By the basic theory of functional differential equations $[6,13]$, we have the following global existence of $(6)$, in which the unknown function is

$$
u=\left(u_{1}^{1}, u_{1}^{2}, \ldots, u_{1}^{m}, u_{2}^{1}, \ldots, u_{n-1}^{m}, u_{n}^{1}, \ldots, u_{n}^{m}\right) .
$$

Lemma 2. Assume that $a_{i}>0, i \in I$. If

$$
v_{i}^{j}(s) \in[0, \infty), s \in[-\tau, 0], i \in I, j \in J,
$$

then (6) has a global solution such that

$$
u_{i}^{j}(t) \in[0, \infty), t>0, i \in I, j \in J .
$$


Moreover, if

$$
\sum_{j \in I} \frac{c_{i j}}{c_{j j}}<2, \quad i \in I
$$

then (6) has a positive steady state

$$
u^{*}=(\overbrace{u_{1}^{*}, \ldots, u_{1}^{*}}^{m}, u_{2}^{*}, \ldots, u_{n-1}^{*}, \overbrace{u_{n}^{*}, \ldots, u_{n}^{*}}^{m}) \gg 0 .
$$

Once the global existence is obtained, we shall prove the following result on the global asymptotic stability of the positive steady state, which also implies the uniqueness of the positive steady state of (6).

Theorem 1. Assume that $a_{i}>0, i \in I$, such that (20) holds and

$$
\sum_{j \in I} \frac{c_{i j}}{\left(c_{j j} a_{j}\right)}<2, \quad i \in I
$$

$$
\left\{\begin{array}{l}
\frac{\mathrm{d} u_{i}^{j}(t)}{\mathrm{d} t} \leq \sum_{l=1}^{m} d_{j l}^{i}\left[\int_{-\tau}^{0} u_{i}^{l}(t+s) d \rho_{j l}^{i}(s) \mathrm{d} s-u_{i}^{j}(t)\right]+r_{i} u_{i}^{j}(t)\left[1-c_{i i} a_{i} u_{i}^{j}(t)\right], \quad t>0 \\
u_{i}^{j}(s)=v_{i}^{j}(s) \geq 0, v_{i}^{j_{0}}(s)>0, \quad \text { for some } j_{0} \in J, j \in J, s \in[-\tau, 0] .
\end{array}\right.
$$

Further consider

$$
\left\{\begin{array}{l}
\frac{\mathrm{d} \bar{u}_{i}^{j}(t)}{\mathrm{d} t}=\sum_{l=1}^{m} d_{j l}^{i}\left[\int_{-\tau}^{0} \bar{u}_{i}^{l}(t+s) d \rho_{j l}^{i}(s) \mathrm{d} s-\bar{u}_{i}^{j}(t)\right]+r_{i} \bar{u}_{i}^{j}(t)\left[1-c_{i i} a_{i} \bar{u}_{i}^{j}(t)\right], \quad t>0, \\
\bar{u}_{i}^{j}(0)=v_{i}^{j}(0) \geq 0, v_{i}^{j_{0}}(0)>0, \quad \text { for some } j_{0} \in J, j \in J, i \in I, s \in[-\tau, 0] .
\end{array}\right.
$$

Then, the comparison principle leads to

$$
\bar{u}_{i}^{j}(t) \geq u_{i}^{j}(t)>0, \quad j \in J, t>0,
$$

since $d_{j l}^{i}>0, j, l \in J$, implies that (29) is a cooperative, irreducible system. By the theory of monotone dynamical systems [6], we have hen

$$
\lim _{t \longrightarrow \infty} u_{i}^{j}(t)=u_{i}^{*}, \quad i \in I, j \in J
$$

In the following, we shall prove seorem 1 by several lemmas. The first is the following result on the positivity of solutions.

Lemma 3. Assume that $a_{i_{0}}>0$ for some $i_{0} \in I$. If

$$
v_{i_{0}}^{j_{0}}(0)>0, \quad \text { for some } j_{0} \in J
$$

then $u_{i_{0}}^{j}(t)>0, t>0, j \in J$, and

$$
\limsup _{t \longrightarrow \infty} u_{i}^{j}(t) \leq\left(c_{i i} a_{i}\right)^{-1}, \quad i=i_{0}, j \in J .
$$

Proof. In the proof, we fix $i=i_{0}$ for simplicity. By $d_{j l}^{i}>0, j, l \in J$, it is clear that $u_{i}^{j}(t)>0, t>0, j \in J$. We now prove (27) by Lemma 2. From the positivity, we have 
After direct calculation, we have the following conclusion by Lemma 1 (we also refer to Smith [6], Lemma 7.4, and Appendix).
Lemma 4. Assume that (24) holds. Define

$$
\begin{aligned}
& a_{i}(y)=y u_{i}^{*}, b_{i}(y)=y u_{i}^{*}+(1-y)\left[\left(c_{i i} a_{i}\right)^{-1}+\varepsilon\right], \quad y \in[0,1], \\
& a(y)=\left(a_{1}(y), a_{2}(y), \ldots, a_{n}(y)\right), b(y)=\left(b_{1}(y), b_{2}(y), \ldots, b_{n}(y)\right), \quad y \in[0,1],
\end{aligned}
$$

with $\varepsilon>0$. If there exists $\varepsilon_{0}>0$ such that

$$
\omega_{i}(s) \in\left(0,\left(c_{i i} a_{i}\right)^{-1}+\varepsilon_{0}\right), s \in[-\tau, 0], i \in I,
$$

and $a(y), b(y)$ with $\varepsilon=\varepsilon_{0}$ satisfy (H3) and (H4) in (32), then

$$
\lim _{t \longrightarrow \infty} w_{i}(t)=u_{i}^{*}, \quad i \in I \text {. }
$$

Lemma 5. Assume that (24) holds and $\varepsilon_{0}>0$ satisfies Lemma 4. Then, there exist $t_{0}>0$ and $y_{0}>0$ such that

$$
u_{i}^{j}\left(t_{0}+s\right) \in\left[a_{i}\left(y_{0}\right), b_{i}\left(y_{0}\right)\right], i \in I, J \in J, s \in[-\tau, 0], \varepsilon=\varepsilon_{0} .
$$

Proof. By (27), we have $t_{0}>\tau$ such that

$$
u_{i}^{j}(t+s)<\left(c_{i i} a_{i}\right)^{-1}+\varepsilon_{0}, \quad i \in I, j \in J, s \in[-\tau, 0], t>t_{0} .
$$

Due to the positivity of solutions in Lemma 3, the result is true by selecting $y_{0}>0$ small enough. The proof is complete.

By what we have done, we now give the proof of Theorem 1 .
Proof. Define

$$
A_{i}^{j}(y)=a_{i}(y), B_{i}^{j}(y)=b_{i}(y), \quad i \in I, j \in J, y \in[0,1],
$$

with $\varepsilon_{0}>0$ small enough. By direct analysis, we can verify that $\left(A_{i}^{j}(y), B_{i}^{j}(y)\right)$ satisfy (H3) and (H4) for (6) if (24) holds (we present the calculation in Appendix). By Lemmas 1 and 5, we complete the proof.

\section{Numerical Simulation}

In this part, we simulate the dynamics of (6) when two patches and three species are involved by selecting some parameters, which may show the plentiful dynamics of such a delayed system without monotonicity. Since $\rho_{j l}^{i}(s)$ in (6) do not change the stability in our results, we shall not consider the delayed effect modeling the spatial movement among different patches.

Example 1. We first try to simulate the dynamics of a system satisfying Theorem 1. For the purpose, we consider the following delayed system:

$$
\left\{\begin{array}{l}
\frac{\mathrm{d} u_{1}^{1}(t)}{\mathrm{d} t}=\left[u_{1}^{2}(t)-u_{1}^{1}(t)\right]+u_{1}^{1}(t)\left[1-u_{1}^{1}(t)-0.2 u_{1}^{1}(t-1)-0.1 u_{2}^{1}(t-1)-0.1 u_{3}^{1}(t-1)\right] \\
\frac{\mathrm{d} u_{1}^{2}(t)}{\mathrm{d} t}=2\left[u_{1}^{1}(t)-u_{1}^{2}(t)\right]+u_{1}^{2}(t)\left[1-u_{1}^{2}(t)-0.2 u_{1}^{2}(t-1)-0.1 u_{2}^{2}(t-1)-0.1 u_{3}^{2}(t-1)\right] \\
\frac{\mathrm{d} u_{2}^{1}(t)}{\mathrm{d} t}=\left[u_{2}^{2}(t)-u_{2}^{1}(t)\right]+u_{2}^{1}(t)\left[1-0.1 u_{1}^{1}(t-1)-u_{2}^{1}(t)-0.1 u_{2}^{1}(t-1)-0.1 u_{3}^{1}(t-1)\right] \\
\frac{\mathrm{d} u_{2}^{2}(t)}{\mathrm{d} t}=2\left[u_{2}^{1}(t)-u_{2}^{2}(t)\right]+u_{2}^{2}(t)\left[1-0.1 u_{1}^{2}(t-1)-u_{2}^{2}(t)-0.1 u_{2}^{2}(t-1)-0.1 u_{3}^{2}(t-1)\right] \\
\frac{\mathrm{d} u_{3}^{1}(t)}{\mathrm{d} t}=\left[u_{3}^{2}(t)-u_{3}^{1}(t)\right]+u_{3}^{1}(t)\left(1-0.3 u_{1}^{1}(t-1)-0.1 u_{2}^{1}(t-1)-u_{3}^{1}(t)-0.1 u_{3}^{1}(t-1)\right) \\
\frac{\mathrm{d} u_{3}^{2}(t)}{\mathrm{d} t}=2\left[u_{3}^{1}(t)-u_{3}^{2}(t)\right]+u_{3}^{2}(t)\left[1-0.3 u_{1}^{2}(t-1)-0.1 u_{2}^{2}(t-1)-u_{3}^{2}(t)-0.1 u_{3}^{2}(t-1)\right]
\end{array}\right.
$$


with $t>0$ and initial condition

$(0.7143,0.7857,0.6429,0.7143,0.7857,0.6429)$.

$$
\begin{aligned}
u_{i}^{1}(s) & =0.05, \quad s \in[-1,0], \\
u_{i}^{2}(s) & =0, \quad s \in[-1,0], \\
i & =1,2,3 .
\end{aligned}
$$

Evidently, (39) satisfies (24) and has a unique positive steady state, of which the approximate value is
From our main result, we see that the positive steady state is asymptotic stable, which is also illustrated in Figure 1.

Example 2. We now consider the possible extinction of some unknown functions when Theorem 1 does not hold and study the dynamics of the following delayed system:

$$
\left\{\begin{array}{l}
\frac{\mathrm{d} u_{1}^{1}(t)}{\mathrm{d} t}=\left[u_{1}^{2}(t)-u_{1}^{1}(t)\right]+u_{1}^{1}(t)\left[1-u_{1}^{1}(t)-0.2 u_{1}^{1}(t-1)-u_{2}^{1}(t-1)-u_{3}^{1}(t-1)\right] \\
\frac{\mathrm{d} u_{1}^{2}(t)}{\mathrm{d} t}=2\left[u_{1}^{1}(t)-u_{1}^{2}(t)\right]+u_{1}^{2}(t)\left[1-u_{1}^{2}(t)-0.2 u_{1}^{2}(t-1)-u_{2}^{2}(t-1)-u_{3}^{2}(t-1)\right] \\
\frac{\mathrm{d} u_{2}^{1}(t)}{\mathrm{d} t}=\left[u_{2}^{2}(t)-u_{2}^{1}(t)\right]+u_{2}^{1}(t)\left[1-0.1 u_{1}^{1}(t-1)-u_{2}^{1}(t)-0.1 u_{2}^{1}(t-1)-0.1 u_{3}^{1}(t-1)\right] \\
\frac{\mathrm{d} u_{2}^{2}(t)}{\mathrm{d} t}=2\left[u_{2}^{1}(t)-u_{2}^{2}(t)\right]+u_{2}^{2}(t)\left[1-0.1 u_{1}^{2}(t-1)-u_{2}^{2}(t)-0.1 u_{2}^{2}(t-1)-0.1 u_{3}^{2}(t-1)\right] \\
\frac{\mathrm{d} u_{3}^{1}(t)}{\mathrm{d} t}=\left[u_{3}^{2}(t)-u_{3}^{1}(t)\right]+u_{3}^{1}(t)\left[1-0.3 u_{1}^{1}(t-1)-0.1 u_{2}^{1}(t-1)-u_{3}^{1}(t)-0.1 u_{3}^{1}(t-1)\right] \\
\frac{\mathrm{d} u_{3}^{2}(t)}{\mathrm{d} t}=2\left[u_{3}^{1}(t)-u_{3}^{2}(t)\right]+u_{3}^{2}(t)\left[1-0.3 u_{1}^{2}(t-1)-0.1 u_{2}^{2}(t-1)-u_{3}^{2}(t)-0.1 u_{3}^{2}(t-1)\right]
\end{array}\right.
$$

with $t>0$ and initial condition (40). Evidently, this system does not satisfy (24), and its dynamics may be different from our Theorem 1, which is presented in Figure 2.
Example 3. The weights among the same species partly play the role of irreducibility, which leads to the possible synchronism. What will happen if the condition does not hold? We consider the dynamics of the following delayed system:

$$
\left\{\begin{array}{l}
\frac{\mathrm{d} u_{1}^{1}(t)}{\mathrm{d} t}=d_{1}\left[u_{1}^{2}(t)-u_{1}^{1}(t)\right]+u_{1}^{1}(t)\left[1-u_{1}^{1}(t)-0.2 u_{1}^{1}(t-1)-0.1 u_{2}^{1}(t-1)-0.1 u_{3}^{1}(t-1)\right] \\
\frac{\mathrm{d} u_{1}^{2}(t)}{\mathrm{d} t}=u_{1}^{2}(t)\left[1-u_{1}^{2}(t)-0.2 u_{1}^{2}(t-1)-0.1 u_{2}^{2}(t-1)-0.1 u_{3}^{2}(t-1)\right], \\
\frac{\mathrm{d} u_{2}^{1}(t)}{\mathrm{d} t}=d_{2}\left[u_{2}^{2}(t)-u_{2}^{1}(t)\right]+u_{2}^{1}(t)\left[1-0.1 u_{1}^{1}(t-1)-u_{2}^{1}(t)-0.1 u_{2}^{1}(t-1)-0.1 u_{3}^{1}(t-1)\right] \\
\frac{\mathrm{d} u_{2}^{2}(t)}{\mathrm{d} t}=u_{2}^{2}(t)\left[1-0.1 u_{1}^{2}(t-1)-u_{2}^{2}(t)-0.1 u_{2}^{2}(t-1)-0.1 u_{3}^{2}(t-1)\right] \\
\frac{\mathrm{d} u_{3}^{1}(t)}{\mathrm{d} t}=d_{3}\left[u_{3}^{2}(t)-u_{3}^{1}(t)\right]+u_{3}^{1}(t)\left[1-0.3 u_{1}^{1}(t-1)-0.1 u_{2}^{1}(t-1)-u_{3}^{1}(t)-0.1 u_{3}^{1}(t-1)\right] \\
\frac{\mathrm{d} u_{3}^{2}(t)}{\mathrm{d} t}=d_{4}\left[u_{3}^{1}(t)-u_{3}^{2}(t)\right]+u_{3}^{2}(t)\left[1-0.3 u_{1}^{2}(t-1)-0.1 u_{2}^{2}(t-1)-u_{3}^{2}(t)-0.1 u_{3}^{2}(t-1)\right]
\end{array}\right.
$$



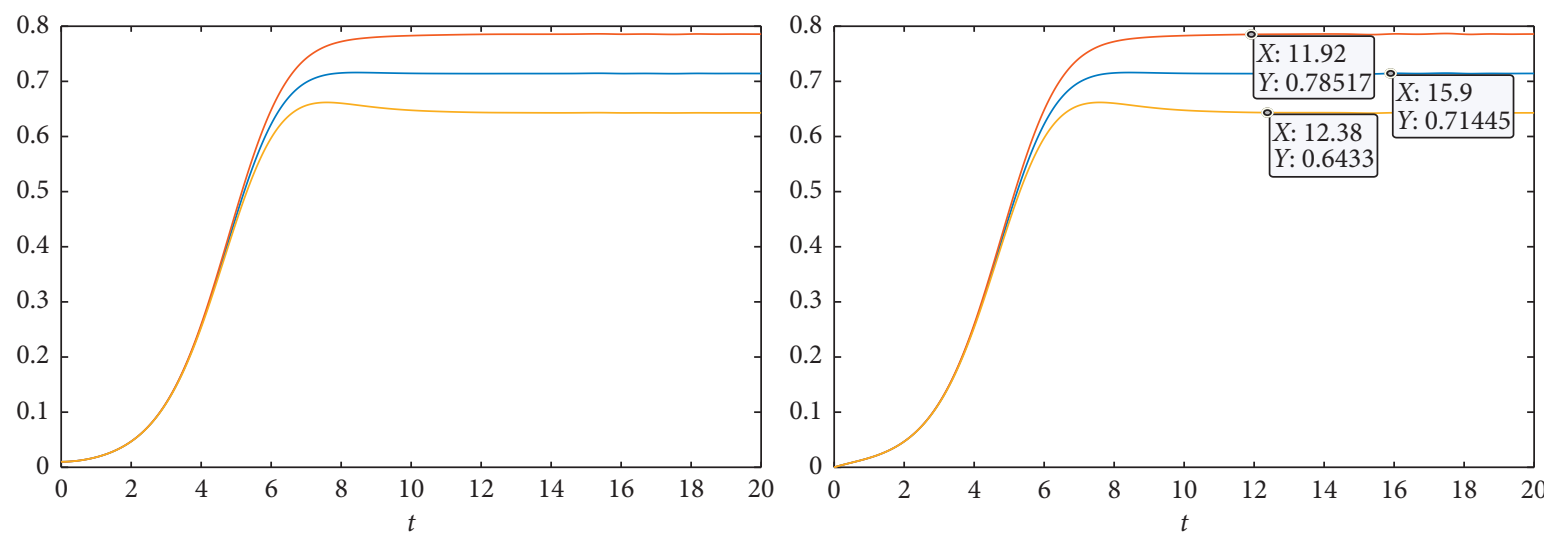

$$
\begin{array}{r}
-u_{1}^{1} \\
-u_{2}^{1} \\
-u_{3}^{1}
\end{array}
$$$$
\begin{array}{r}
-u_{1}^{2} \\
-u_{2}^{2} \\
-u_{3}^{2}
\end{array}
$$

FIGURE 1: Simulation of (39) with (40).

with $t>0$ and initial condition (40), and $d_{1}, \ldots, d_{4}$ are positive.

In this system, the weights may be zero, and it does not satisfy our condition. By simulation in Figures 3 and 4, we may observe different persistence-extinction phenomena with partially degenerate weights.
Example 4. Finally, we try to explore the influence of delay size. In the literature, some nontrivial results of the delay size have been reported even in scalar equations [2]. We consider the dynamics of the following delayed system:

$$
\left\{\begin{array}{l}
\frac{\mathrm{d} u_{1}^{1}(t)}{\mathrm{d} t}=\left[u_{1}^{2}(t)-u_{1}^{1}(t)\right]+u_{1}^{1}(t)\left[1-0.2 u_{1}^{1}(t)-u_{1}^{1}(t-\tau)-0.1 u_{2}^{1}(t-\tau)-0.1 u_{3}^{1}(t-\tau)\right] \\
\frac{\mathrm{d} u_{1}^{2}(t)}{\mathrm{d} t}=2\left[u_{1}^{1}(t)-u_{1}^{2}(t)\right]+u_{1}^{2}(t)\left[1-0.2 u_{1}^{2}(t)-u_{1}^{2}(t-\tau)-0.1 u_{2}^{2}(t-\tau)-0.1 u_{3}^{2}(t-\tau)\right] \\
\frac{\mathrm{d} u_{2}^{1}(t)}{\mathrm{d} t}=\left[u_{2}^{2}(t)-u_{2}^{1}(t)\right]+u_{2}^{1}(t)\left[1-0.1 u_{1}^{1}(t-\tau)-0.1 u_{2}^{1}(t)-u_{2}^{1}(t-\tau)-0.1 u_{3}^{1}(t-\tau)\right] \\
\frac{\mathrm{d} u_{2}^{2}(t)}{\mathrm{d} t}=2\left[u_{2}^{1}(t)-u_{2}^{2}(t)\right]+u_{2}^{2}(t)\left[1-0.1 u_{1}^{2}(t-\tau)-0.1 u_{2}^{2}(t)-u_{2}^{2}(t-\tau)-0.1 u_{3}^{2}(t-\tau)\right] \\
\frac{\mathrm{d} u_{3}^{1}(t)}{\mathrm{d} t}=\left[u_{3}^{2}(t)-u_{3}^{1}(t)\right]+u_{3}^{1}(t)\left[1-0.3 u_{1}^{1}(t-\tau)-0.1 u_{2}^{1}(t-\tau)-0.1 u_{3}^{1}(t)-u_{3}^{1}(t-\tau)\right] \\
\frac{\mathrm{d} u_{3}^{2}(t)}{\mathrm{d} t}=2\left[u_{3}^{1}(t)-u_{3}^{2}(t)\right]+u_{3}^{2}(t)\left[1-0.3 u_{1}^{2}(t-\tau)-0.1 u_{2}^{2}(t-\tau)-0.1 u_{3}^{2}(t)-u_{3}^{2}(t-\tau)\right]
\end{array}\right.
$$

with $\tau>0, t>0$, and initial value condition

$$
\begin{aligned}
& u_{1}(s)=u_{2}(s)=u_{3}(s)=0.05, \quad s \in[-\tau, 0] \\
& u_{4}(s)=u_{5}(s)=u_{6}(s)=0, \quad s \in[-\tau, 0] .
\end{aligned}
$$

By our simulations in Figure 5, we find that large delay may lead to the instability of the positive steady state, while the small delay may be harmless to the stability of the positive steady state.

\section{Discussion}

In this paper, we studied a delayed model on a weighted network, which may be regarded as a delayed competition model on patches. We established the global stability of the 

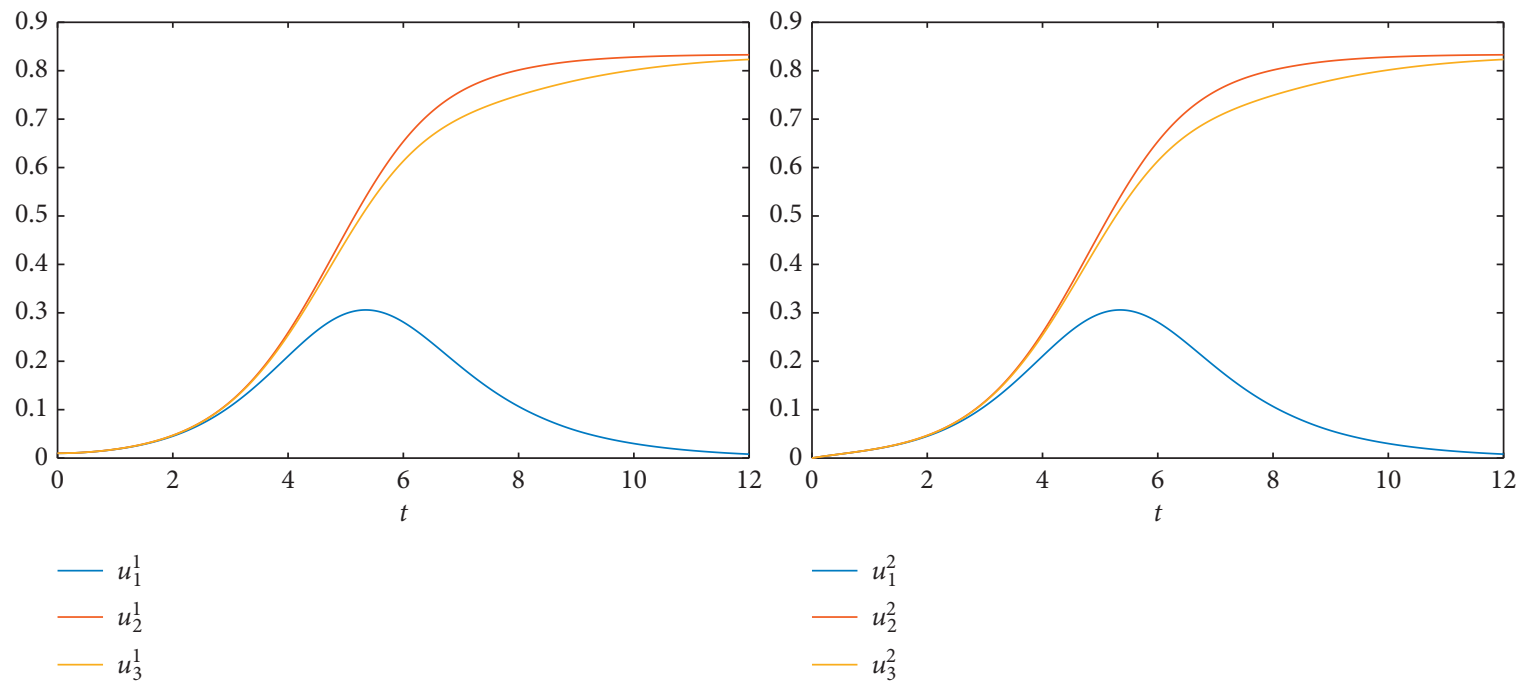

Figure 2: Simulation of (42) with (40).
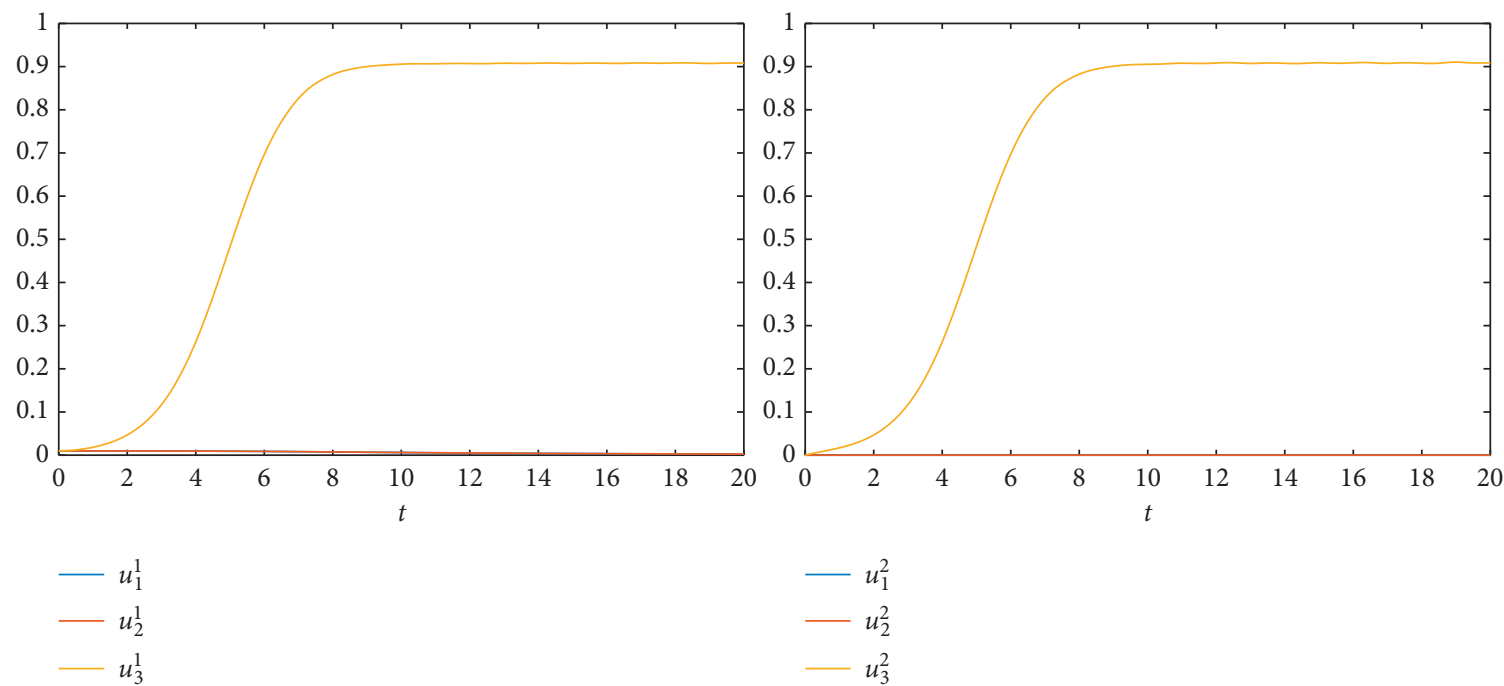

Figure 3: Simulation of (43) with (40) by fixing $\left(d_{1}, d_{2}, d_{3}, d_{4}\right)=(1,1,1,2)$.

unique positive steady state by constructing contracting rectangles under condition (24). Moreover, we gave some numerical examples to model the dynamics of (6). From Examples 1-4, we found that the dynamics of this nonmonotone system may be very complex, which includes the stability of different steady states. In particular, if the weight coefficients among some patches are zero, the uniform convergence on different patches may be false even if (24) holds, and we observed this from Example 2. That is, weights among different patches may affect the dynamics of this system, and the role of weights should be further studied.

Moreover, if (24) does not hold, then the stability of the positive steady state may depend on the size of time delay, which is partially motivated by the dynamics of the classical delayed logistic model [27]. From Example 4, we observed different convergence results with different delays. To further show the effect of time delay in such a delayed model is an interesting question. However, with the increase in the number of unknown functions, the analysis of eigenvalues becomes complex, and this is a challenging question. We shall further consider these questions in our future works.

Finally, in many other mathematical models including uncertain discrete-time descriptor systems with multiple time-varying delays and parametric uncertainties [28], nonholonomic systems with time-varying delays and nonlinear disturbances [29], and uncertain chaotic systems with nonlinearity and time delay [30], it is important to estimate the dynamics in finite time. Due to the effect of time-varying delays, this is not a trivial work and deserves further investigation. Because the contracting rectangles could reflect certain uniform convergence, it is possible to consider the finite-time dynamics of these important models by contracting rectangles, at least when the initial data satisfy proper uniform conditions in the delayed interval. 

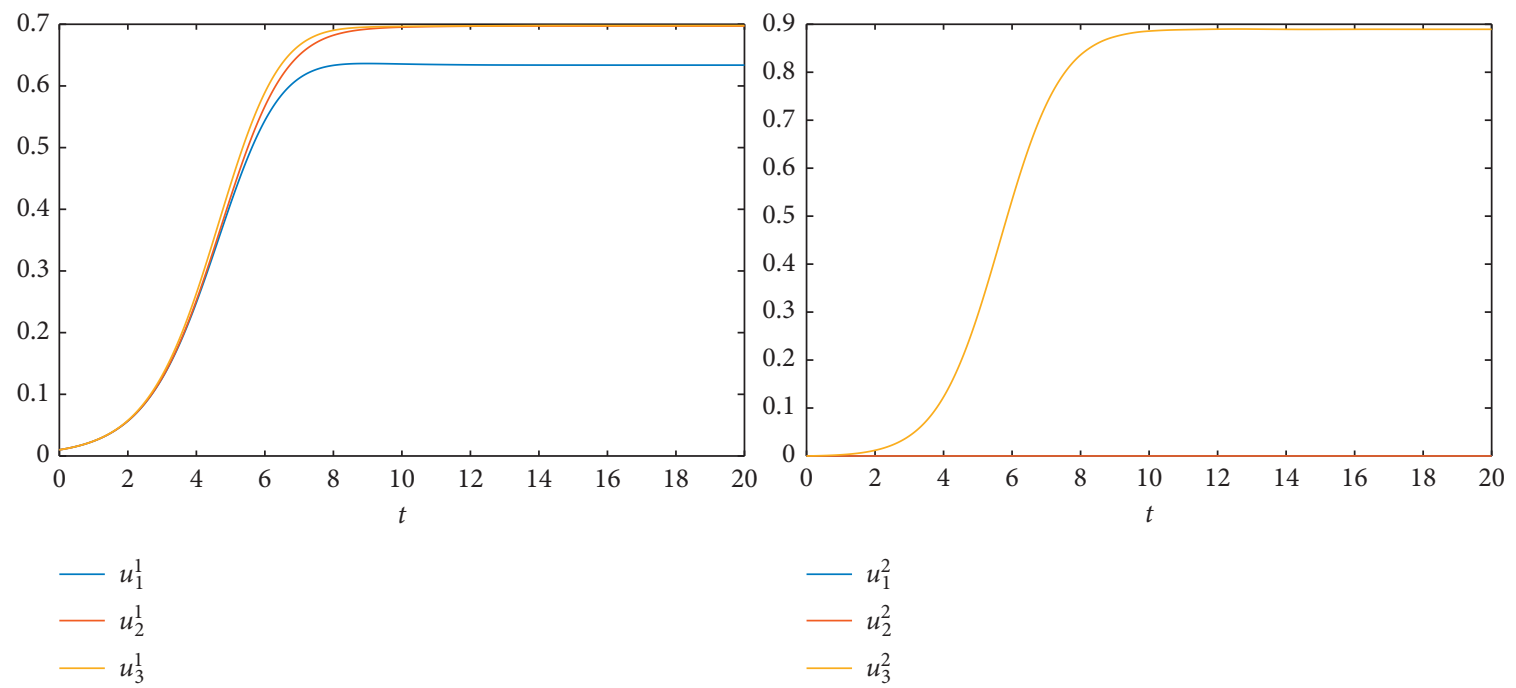

Figure 4: Simulation of (43) with (40) by fixing $\left(d_{1}, d_{2}, d_{3}, d_{4}\right)=(0.1,0.1,0.1,0.1)$.
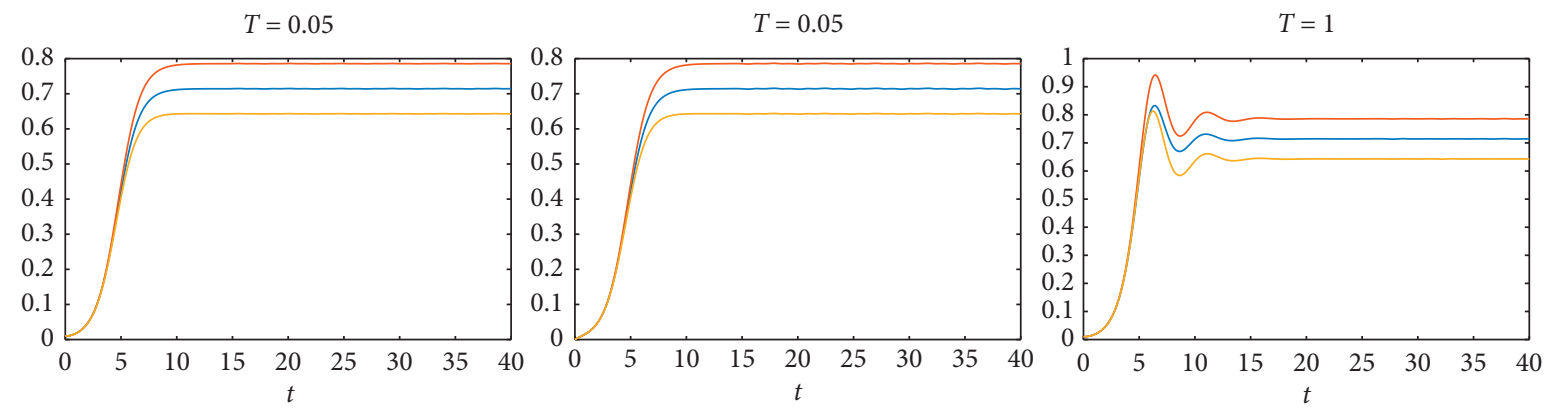

$$
\begin{array}{r}
-u_{1}^{1} \\
-u_{2} \\
-u_{3}^{1}
\end{array}
$$

$-u_{1}^{2}$

$-u_{2}^{2}$

$-u_{3}^{2}$

$$
\begin{aligned}
& -u_{1}^{1} \\
& -u_{2}^{1} \\
& -u_{3}^{1}
\end{aligned}
$$
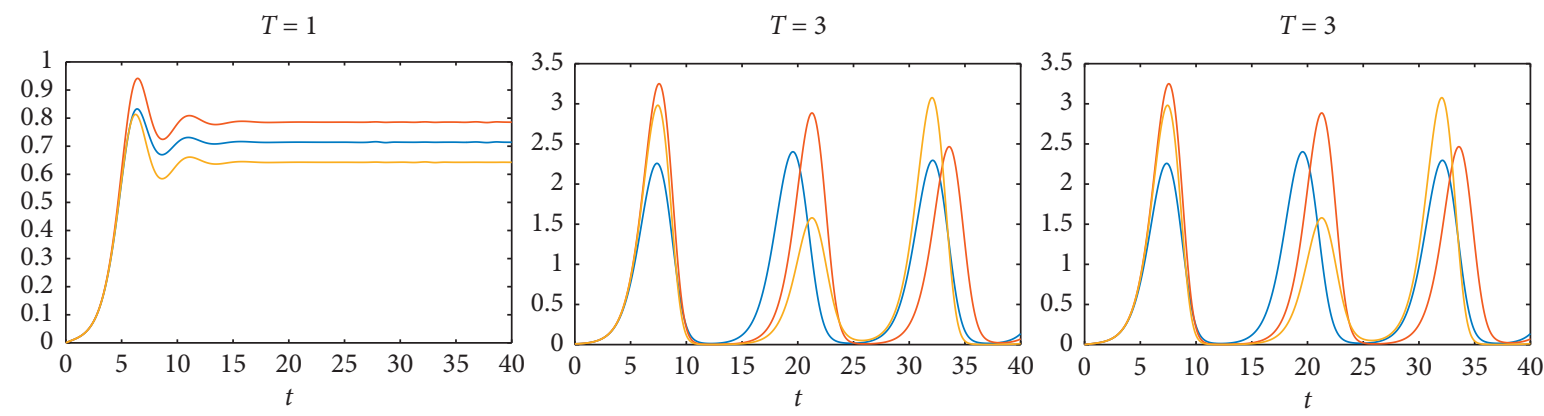

$$
\begin{array}{r}
-u_{1}^{2} \\
-u_{2}^{2} \\
-u_{3}^{2}
\end{array}
$$
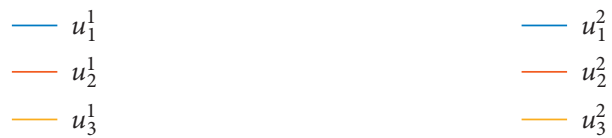

FIGURE 5: Simulation of (44) with (45) by taking different time delays. 


\section{Appendix}

In this part, we verify the definition of contracting rectangles in (6) and (32). Since the verification of (32) can be obtained by that of (6) and can be found in [6], we only verify the result on (6).

We first verify the conclusion on $A_{i}^{j}(y)$ in (H4); if $u_{i}^{j}(t)=A_{i}^{j}(y), y \in(0,1)$, and

$$
u_{k}^{l}(t+s) \in\left[A_{i}^{j}(y), B_{i}^{j}(y)\right], k \in I, j \in J, s \in[-\tau, 0],
$$

for some $t \geq 0$, then

$$
1-\sum_{k=1}^{n} \frac{c_{i k}}{c_{k k} a_{k}} \int_{-\tau}^{0} \mathrm{~d} \eta_{i k}(s)>0
$$

by selecting $\varepsilon>0$ small enough since (24) implies

$$
1-\sum_{k=1}^{n} \frac{c_{i k}}{c_{k k} a_{k}} \int_{-\tau}^{0} \mathrm{~d} \eta_{i k}(s)>0,
$$

which also implies the admissibility of $\varepsilon_{0}>0$ in the proof of Theorem 1.

We now verify the conclusion on $B_{i}^{j}(y)$ in (H4); if $u_{i}^{j}(t)=B_{i}^{j}(y), y \in(0,1)$, and

$$
u_{k}^{l}(t+s) \in\left[A_{i}^{j}(y), B_{i}^{j}(y)\right], k \in I, j \in J, s \in[-\tau, 0],
$$

for some $t>0$, then

$$
\begin{aligned}
& \sum_{l=1}^{m} d_{j l}^{i}\left[\int_{-\tau}^{0} u_{i}^{l}(t+s) d \rho_{j l}^{i}(s) \mathrm{d} s-u_{i}^{j}(t)\right]+r_{i} u_{i}^{j}(t)\left[1-\sum_{k=1}^{n} c_{i k} \int_{-\tau}^{0} u_{k}^{j}(t+s) \mathrm{d} \bar{\eta}_{i k}(s)\right] \\
& =\sum_{l=1}^{m} d_{j l}^{i}\left[\int_{-\tau}^{0} u_{i}^{l}(t+s) d \rho_{j l}^{i}(s) \mathrm{d} s-u_{i}^{j}(t)\right]+r_{i} u_{i}^{j}(t)\left[1-c_{i i} a_{i} u_{i}^{j}(t)-\sum_{k=1}^{n} c_{i k} \int_{-\tau}^{0} u_{k}^{j}(t+s) \mathrm{d} \eta_{i k}(s)\right] \\
& =\sum_{l=1}^{m} d_{j l}^{i}\left[\int_{-\tau}^{0} u_{i}^{l}(t+s) d \rho_{j l}^{i}(s) \mathrm{d} s-u_{i}^{j}(t)\right]+r_{i} B_{i}^{j}(y)\left[1-c_{i i} a_{i} B_{i}^{j}(y)-\sum_{k=1}^{n} c_{i k} \int_{-\tau}^{0} u_{k}^{j}(t+s) \mathrm{d} \eta_{i k}(s)\right] \\
& \leq r_{i} B_{i}^{j}(y)\left[1-c_{i i} a_{i} B_{i}^{j}(y)-\sum_{k=1}^{n} c_{i k} \int_{-\tau}^{0} u_{k}^{j}(t+s) \mathrm{d} \eta_{i k}(s)\right] r_{i} B_{i}^{j}(y)\left[1-c_{i i} a_{i} B_{i}^{j}(y)-\sum_{k=1}^{n} c_{i k} \int_{-\tau}^{0} A_{k}^{j} \mathrm{~d} \eta_{i k}(s)\right] \\
& =r_{i} B_{i}^{j}(y)\left[1-c_{i i} a_{i}\left[y u_{i}^{*}+(1-y)\left[\left(c_{i i} a_{i}\right)^{-1}+\varepsilon\right]\right]-\sum_{k=1}^{n} c_{i k} \int_{-\tau}^{0} y u_{k}^{*} \mathrm{~d} \eta_{i k}(s)\right] \\
& =r_{i} B_{i}^{j}(y)\left[y-c_{i i} a_{i} y u_{i}^{*}-\sum_{k=1}^{n} c_{i k} \int_{-\tau}^{0} y u_{k}^{*} \mathrm{~d} \eta_{i k}(s)\right]+r_{i} B_{i}^{j}(y)\left[1-y-(1-y) c_{i i} a_{i}\left[\left(c_{i i} a_{i}\right)^{-1}+\varepsilon\right]\right] \\
& =r_{i}(1-y) B_{i}^{j}(y)\left[1-c_{i i} a_{i}\left[\left(c_{i i} a_{i}\right)^{-1}+\varepsilon\right]\right] \\
& <0
\end{aligned}
$$

by $\varepsilon>0$. Due to the arbitrary of $i$ and $j$, we finish the verification.

\section{Data Availability}

The data used to support the findings of this study are included within the article.

\section{Conflicts of Interest}

The authors declare that there are no conflicts of interest regarding the publication of this paper.

\section{Authors' Contributions}

All authors contributed equally to the writing of this paper. All authors read and approved the final manuscript.

\section{References}

[1] J. D. Murray, "Mathematical biology, I. an introduction," Interdisciplinary Applied Mathematics, Vol. 18, SpringerVerlag, New York, NY, USA, Third edition, 2002.

[2] J. K. Hale and S. M. Verduyn Lunel, Introduction to Functional-Differential Equations, Springer-Verlag, New York, NY, USA, 1993.

[3] Y. Kuang, "Delay differential equations with applications in population dynamics," Mathematics in Science and Engineering, vol. 191, 1993.

[4] J. Wu, Theory and Applications of Partial Functional Differential Equations, Springer-Verlag, New York, NY, USA, 1996.

[5] T. Faria, "Global dynamics for Lotka-Volterra systems with infinite delay and patch structure," Applied Mathematics and Computation, vol. 245, pp. 575-590, 2014.

[6] H. L. Smith, Monotone Dynamical Systems: An Introduction to the Theory of Competitive and Cooperative Systems, AMS, Providence, RI, USA, 1995. 
[7] N. F. Britton, Reaction-diffusion Equations and their Applications to Biology, Academic Press, London, UK, 1986.

[8] R. S. Cantrell and C. Cosner, Spatial Ecology via ReactionDiffusion Equations, John Wiley \& Sons, Ltd., Boca Raton, Florida, 2003.

[9] J. Smoller, Shock Waves and Reaction Diffusion Equations, Springer-Verlag, New York, NY, USA, 1994.

[10] Q. Ye, Z. Li, M. Wang, and Y. Wu, Introduction to Reaction Diffusion Equations, Science Press, Beijing, China, Second edition, 2011.

[11] X. He and W. M. Ni, "Global dynamics of the Lotka-Volterra competition-diffusion system: diffusion and spatial heterogeneity I," Communications on Pure and Applied Mathematics, vol. 69, pp. 981-1014, 2006.

[12] H. F. Weinberger, M. A. Lewis, and B. Li, "Analysis of linear determinacy for spread in cooperative models," Journal of Mathematical Biology, vol. 45, no. 3, pp. 183-218, 2002.

[13] R. H. Martin and H. L. Smith, "Reaction-diffusion systems with the time delay: monotonicity, invariance, comparison and convergence," Journal Für Die Reine Und Angewandte Mathematik, vol. 413, pp. 1-35, 1991.

[14] E. Beretta, F. Solimano, and Y. Takeuchi, "Global stability and periodic orbits for two-patch predator-prey diffusion-delay models," Mathematical Biosciences, vol. 85, no. 2, pp. 153-183, 1987.

[15] D. Gao and S. Ruan, "A multipatch Malaria model with logistic growth populations," SIAM Journal on Applied Mathematics, vol. 72, no. 3, pp. 819-841, 2012.

[16] K.-L. Liao and Y. Lou, "The effect of time delay in a two-patch model with random dispersal," Bulletin of Mathematical Biology, vol. 76, no. 2, pp. 335-376, 2014.

[17] A. Mai, G. Sun, and L. Wang, "The impacts of dispersal on the competition outcome of multi-patch competition models," Mathematical Biosciences and Engineering, vol. 16, no. 4, pp. 2697-2716, 2019.

[18] G. Sun and A. Mai, "Stability analysis of a two-patch competition model with dispersal delays," Discrete Dynamics in Nature and Society, vol. 2019, p. 6, Article ID 3159591, 2019.

[19] R. Xu and L. Chen, "Global stability for a generalized predator-prey system with time delay in two-patch environments," Applicable Analysis, vol. 82, pp. 677-687, 2003.

[20] Z. Zhang and Z. Wang, "Periodic solution for a two-species nonautonomous competition Lotka-Volterra patch system with time delay," Journal of Mathematical Analysis and Applications, vol. 265, no. 1, pp. 38-48, 2002.

[21] Z. Liu, C. Tian, and S. Ruan, "On a network model of two competitors with applications to the invasion and competition of Aedes albopictus and Aedes aegypti mosquitoes in the United States," SIAM Journal on Applied Mathematics, vol. 80, no. 2, pp. 929-950, 2020.

[22] M. Cai and Z. Xiang, "Adaptive neural finite-time control for a class of switched nonlinear systems," Neurocomputing, vol. 155, pp. 177-185, 2015.

[23] Y. Chang, S. Zhang, N. D. Alotaibi, and A. F. Alkhateeb, "Observer-based adaptive finite-time tracking control for a class of switched nonlinear systems with unmodeled dynamics," IEEE Access, vol. 8, pp. 204782-204790, 2020.

[24] J. Mao, Z. Xiang, and S. Huang, "Adaptive finite-time tracking control for a class of switched nonlinear systems with unmodeled dynamics," Neurocomputing, vol. 196, pp. 42-52, 2016.

[25] R. A. Armstrong and R. McGehee, "Coexistence of species competing for shared resources," Theoretical Population Biology, vol. 9, no. 3, pp. 317-328, 1976.
[26] P. Chesson, "General theory of competitive coexistence in spatially-varying environments," Theoretical Population Biology, vol. 58, no. 3, pp. 211-237, 2000.

[27] S. Ruan, "Delay differential equations," in Delay Differential Equations with Applications, NATO Science Series II: Mathematics, Physics and Chemistry, O. Arino, M. Hbid, and E. Ait Dads, Eds., pp. 477-517, Springer-Verlag, Berlin, Germany, 2002.

[28] S. Mobayen, F. Bayat, H. Omidvar, and A. Fekih, "Robust global controller design for discrete-time descriptor systems with multiple time-varying delays," International Journal of Robust and Nonlinear Control, vol. 30, no. 7, pp. 2809-2831, 2020.

[29] S. Rasoolinasab, S. Mobayen, A. Fekih, P. Narayan, and Y. Yao, "A composite feedback approach to stabilize nonholonomic systems with time varying time delays and nonlinear disturbances," ISA Transactions, vol. 101, pp. 177-188, 2020.

[30] S. Mobayen and J. Ma, "Robust finite-time composite nonlinear feedback control for synchronization of uncertain chaotic systems with nonlinearity and time-delay," Chaos, Solitons \& Fractals, vol. 114, pp. 46-54, 2018. 